\title{
Dupuytren's contractures associated with the BRAF inhibitor vemurafenib: a case report
}

\author{
Sze Wai Chan ${ }^{*}$ and Daniel Alberto Vorobiof
}

\begin{abstract}
Introduction: Two previous cases of the development of Dupuytren's contractures were reported in association with BRAF inhibitor treatment for BRAF V600E mutation-positive metastatic melanoma and metastatic papillary thyroid carcinoma. We reported on a third case with a slower onset of presentation.

Case presentation: A 66-year-old white man was diagnosed with a BRAF V600E mutated metastatic cutaneous melanoma. He was commenced on oral vemurafenib 960mg twice daily. A marked response was achieved for his metastatic disease. He noticed a change of his hair characteristics and a feeling of "lumps" in both palms by 6 months. By 9 months, classical Dupuytren's contracture was apparent.

Conclusions: Dupuytren's contracture is not a known side effect of BRAF inhibitor treatment. The timeline for the development of Dupuytren's contracture on BRAF inhibitor treatment is not well defined. Although the etiology of Dupuytren's contracture is unknown, an increase in tumor necrosis factor has been demonstrated to be a possible mechanism. BRAF inhibition has been shown to increase immune reaction in the tumor microenvironment and is associated with high serum tumor necrosis factor level. We propose that an increased level of tumor necrosis factor associated with BRAF inhibition may increase the risk of the development of Dupuytren's contractures.
\end{abstract}

Keywords: BRAF V600 mutation, Cutaneous malignant melanoma, Dupuytren's contracture, Vemurafenib

\section{Introduction}

Vemurafenib, an oral anti-BRAF V600 kinase inhibitor, is indicated for the treatment of advanced malignant melanoma for patients whose tumors harbor the BRAF V600 mutation. Vemurafenib inhibits the MAP kinase pathway by binding to the kinase domain in mutant BRAF and has been shown to prolong both progression free and overall survival [1].

Toxicity from vemurafenib is common and includes many cutaneous side effects (skin rash, photosensitivity, hyperkeratosis, cutaneous squamous cell carcinoma, keratoacanthoma, and skin papilloma), alopecia, arthralgia, headache, fatigue, diarrhea and nausea [2-4].

Recently two cases of Dupuytren's contractures have been reported in the medical literature in patients receiving a BRAF inhibitor $[5,6]$. We report on an additional case, different in development when compared to the cases previously published.

\footnotetext{
* Correspondence: dr.szewai.chan@gmail.com

Sandton Oncology Centre, 159 Rivonia Road, Morningside, Sandton, Johannesburg, Gauteng 2199, South Africa
}

\section{Case presentation}

A 66-year-old white man was diagnosed with a BRAF V600E mutated metastatic cutaneous melanoma with subcutaneous metastases. He was known to have asthma for which he needed salbutamol and fluticasone inhalers. He had no other medical history of note. He was enrolled onto a national clinical trial and after signing an informed consent he was commenced on oral vemurafenib 960mg twice daily. A marked response was achieved (complete response) and his metastatic subcutaneous lesion disappeared after 5 months. He experienced grade 1 side effects such as arthralgia, a macular non-itchy skin rash over his upper chest, photosensitivity in sun exposed areas and general malaise. The appearance of hyperkeratotic lesions, keratoacanthomas and one basal cell carcinoma were treated with excisions, without a need to change his planned treatment dose.

Approximately 6 months after the start of vemurafenib treatment, he noticed a change in his hair characteristics to curly hair (Fig. 1) and he started to feel "lumps" in both of his palms. By 9 months, most of his skin rash 


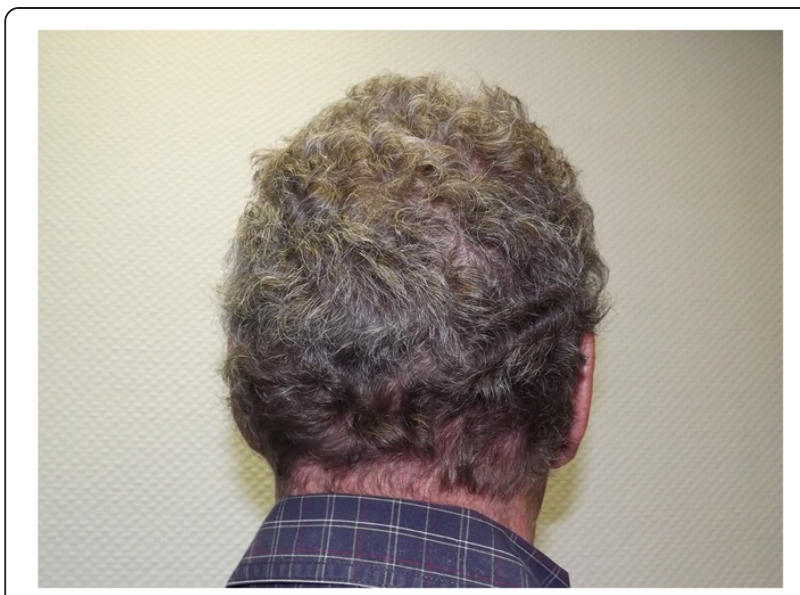

Fig. 1 Development of curly hair on vemurafenib treatment

had disappeared and the "lumps" in his hands became noticeable and harder.

A clinical examination demonstrated painless nodules in both palms and formation of a fibrous band proximal to his 4th and 5th digits, consistent with a diagnosis of Dupuytren's contractures (Fig. 2). There was no functional impairment with finger extension.

As he remained asymptomatic, a watch and wait approach was adopted with continuation of vemurafenib therapy.

\section{Discussion}

Dupuytren's contracture is a benign, slowly progressive fibrosis of the palmar fascia. It is a result of fibroblastic proliferation and disorderly collagen deposition. The

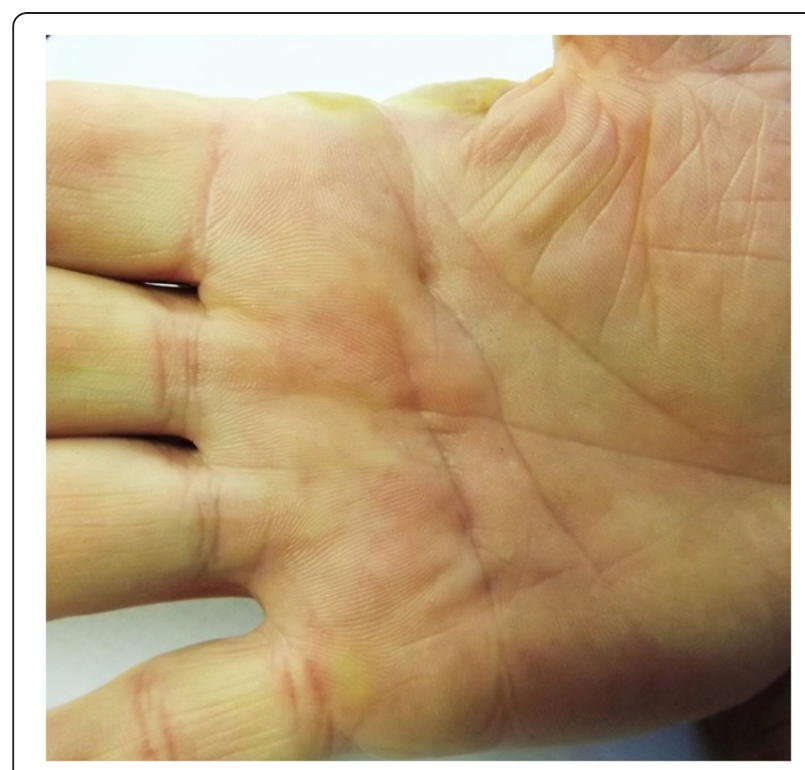

Fig. 2 Development of fibrous band and palmar nodules suggestive of Dupuytren's contractures early proliferative stage is associated with painless or painful nodules in the palms. With continued fibrosis, it will progress to form longitudinal bands or cords, limiting finger extension. Thumb and index fingers are usually spared and 4th and 5th fingers are commonly affected.

The etiology of Dupuytren's contracture is unknown. Most patients present over the age of 50; it is more common in relatives of affected patients, male gender and people of European descent [7, 8]. There was no definitive association with a history of cigarette smoking, alcohol consumption or repetitive handling tasks. Our patient's occupation was office-based and he enjoys gardening, fishing and golf.

On review of the current available medical literature, the first reported case of Dupuytren's contractures secondary to BRAF kinase inhibitor therapy was reported by Bicknell et al. [5]. The described patient had a BRAF mutation-positive metastatic papillary thyroid carcinoma and was treated with a BRAF inhibitor on a clinical trial and developed Dupuytren's contractures of both hands, palmar hyperkeratosis, a keratosis pilaris-like eruption and erythema nodosum [5].

Our patient presented with classical features of Dupuytren's contractures such as palmar nodules and fibrous band formation, of a slow onset, manifesting at 6 months after commencement of vemurafenib treatment. He had neither flexion contractures nor functional impairment and his dosage of vemurafenib was not interrupted or discontinued.

This is also very different from a patient described by Sibaud and Chevreau where there was an abrupt onset of Dupuytren's contractures after 6 weeks of vemurafenib treatment for metastatic melanoma with metastatic cervical lymph nodes [6]. The patient had a rapid development of fixed digital flexion contractures and visible cords but no associated palmar nodules. Discontinuation of vemurafenib was necessary due to the rapidly progressive nature and functional impairment affecting activities of daily living. There was however, no improvement or deterioration at 3 months after discontinuation of vemurafenib [6].

Dupuytren's contracture is not a known side effect resulting from the BRAF kinase inhibitor vemurafenib. Sibaud and Chevreau hypothesized that vemurafenib induces a paradoxical proliferation in wild type-BRAF myofibroblasts [6]. BRAF inhibition has been shown to increase immune reaction in the tumor microenvironment. Wilmott et al. demonstrated that BRAF inhibitortreated patients with metastatic melanoma have higher serum levels of interferon- $\gamma$ (IFN- $\gamma)$, CCL4, and tumor necrosis factor (TNF) [9]. Verjee et al. have shown that significant numbers of immune cells and a high level of pro-inflammatory cytokines are found in Dupuytren's 
tissue where TNF selectively converted normal fibroblasts from the palms of patients with Dupuytren's contractures into myofibroblasts via activation of the Wnt signaling pathway. Neutralizing antibodies to TNF reverse this phenomenon, suggesting that TNF inhibition may prevent progression or recurrence of Dupuytren's contractures [10].

We propose that an increased level of TNF associated with BRAF inhibition may increase the risk of the development of Dupuytren's contractures.

\section{Conclusions}

The slow onset of the development of Dupuytren's contracture in our patient suggested that the timeline for the development of Dupuytren's contracture is not well defined on BRAF inhibitor treatment. An increased level of TNF associated with BRAF inhibition may increase the risk of the development of Dupuytren's contractures. We need to be vigilant on this rare side effect throughout the course of the BRAF inhibitor treatment.

\section{Consent}

Written informed consent was obtained from the patient for publication of this case report and any accompanying images. A copy of the written consent is available for review by the Editor-in-Chief of this journal.

\section{Competing interests}

The authors declare that they have no competing interests.

\section{Authors' contributions}

SC carried out the informed consent and photography, drafted the manuscript and carried out the literature search and review. DV carried out the informed consent, participated in the drafting and editing of the manuscript and he is the treating physician of the patient in this case report. All authors read and approved the final manuscript.

\section{Authors' information}

SC and DV are medical oncologists in private practice at Sandton Oncology Centre, Johannesburg, South Africa.

SC and DV have a special interest in the latest advances in the treatment of malignant melanoma.

\section{Acknowledgements}

We would like to acknowledge and thank the patient for participating in this case report.

Received: 3 November 2014 Accepted: 15 June 2015

Published online: 08 July 2015

\section{References}

1. Chapman PB, Hauschild A, Robert C, Haanen JB, Ascierto P, Larkin J, et al. Improved survival with vemurafenib in melanoma with BRAF V600E mutation. N Engl J Med. 2011:364:2507-16.

2. McArthur GA, Chapman PB, Robert C, Larkin J, Haanen JB, Dummer R, et al. Safety and efficacy of vemurafenib in BRAF(V600E) and BRAF(V600K) mutation-positive melanoma (BRIM-3): extended follow-up of a phase, 3, randomised, open-label study. Lancet Oncol. 2014:15:323-32.

3. Larkin J, Del Vecchio M, Ascierto PA, Krajsova I, Schachter J, Neyns B, et al. Vemurafenib in patients with BRAF(V600) mutated metastatic melanoma: an open-label, multicentre, safety study. Lancet Oncol. 2014;15:436-44.
4. Lacouture ME, Duvic M, Hauschild A, Prieto VG, Robert C, Schadendorf D, et al. Analysis of dermatologic events in vemurafenib-treated patients with melanoma. Oncologist. 2013;18:314-22.

5. Bicknell LTM, Ciurea A, Diaz L, Hymes S. Dupuytren's contractures secondary to BRAF kinase inhibitor therapy. J Am Acad Dermatol. 2013;68:AB179.

6. Sibaud V, Chevreau C. Abrupt development of Dupuytren's contractures with the BRAF inhibitor vemurafenib. Joint Bone Spine. 2014;81:373-4.

7. Ross DC. Epidemiology of Dupuytren's disease. Hand Clin. 1999;15:53-62.

8. Anthony SG, Lozano-Calderon SA, Simmons BP, Jupiter JB. Gender ratio of Dupuytren's disease in the modern U.S. population. Hand (N Y). 2008;3:87-90

9. Wilmott JS, Haydu LE, Am M, Lum T, Hyman J, Thompson JF, et al. Dynamics of chemokine, cytokine, and growth factor serum levels in BRAF-mutant melanoma patients during BRAF inhibitor treatment. J Immunol. 2014;192:2505-13.

10. Verjee LS, Verhoekx JS, Chan JK, Krausgruber T, Nicolaidou V, Izadi D, et al. Unraveling the signaling pathways promoting fibrosis in Dupuytren's disease reveals TNF as a therapeutic target. Proc Natl Acad Sci U S A. 2013;110:E928-37.

\section{Submit your next manuscript to BioMed Central and take full advantage of:}

- Convenient online submission

- Thorough peer review

- No space constraints or color figure charges

- Immediate publication on acceptance

- Inclusion in PubMed, CAS, Scopus and Google Scholar

- Research which is freely available for redistribution 